\title{
Solving a bi-objective mathematical programming model for bloodmobiles location routing problem
}

\author{
Masoud Rabbani $^{\mathrm{a}^{*}}$, Mohsen Aghabegloo a and Hamed Farrokhi-Asl ${ }^{\mathrm{b}}$ \\ ${ }^{a}$ School of Industrial Engineering, College of Engineering, University of Tehran, Tehran, Iran \\ ${ }^{b}$ School of Industrial Engineering, Iran University of Science \& Technology, Tehran, Iran \\ C H R O N I C L E \\ Article history: \\ Received Februray 42016 \\ Received in Revised Format \\ June 162016 \\ Accepted July 282016 \\ Available online \\ July 312016 \\ Keywords: \\ Vehicle routing problem \\ Bloodmobiles \\ Simulated annealing \\ Fuzzy multi objective \\ programming \begin{abstract}
A B S T R A C T
Perishability of platelets, uncertainty of donors' arrival and conflicting views in platelet supply chain have made platelet supply chain planning a problematic issue. In this paper, mobile blood collection system for platelet production is investigated. Two mathematical models are presented to cover the bloodmobile collection planning problem. The first model is a multi-objective fuzzy mathematical programming in which the bloodmobiles locations are considered with the aim of maximizing potential amount of blood collection and minimizing the operational cost. The second model is a vehicle routing problem with time windows which studies the shuttles routing problem. To tackle the first model, it is reformulated as a crisp multi objective linear programming model and then solved through a fuzzy multi objective programming approach. Several sensitivity analysis are conducted on important parameters to demonstrate the applicability of the proposed model. The proposed model is then solved by using a tailored Simulated Annealing (SA) algorithm. The numerical results demonstrate promising efficiency of the proposed solution method.
\end{abstract}

\section{Introduction}

Healthcare supply chain plays a key role in efficiency, efficacy, and reliability of healthcare systems. In addition, a great portion of healthcare costs relates to healthcare supply chain. Therefore, different operations in healthcare supply chain have been widely recognized among both practitioners and academicians. One of the key products which has been widely studied in this regard, is human blood. Specific characteristics of blood products, stochastic behaviors existed in demand and supply zones, as well as criticality of blood supplementing for human beings have made the blood supply chain an interest issue for practitioners, academicians, and governments. Patients severing from injuries, variety of diseases, or having surgery need blood transfusion. It is stated that "every two seconds someone in U.S needs blood and more than 41,000 blood donations are needed every day" (Rezaei-Malek et al., 2016). This leads hospitals to blood supply chain issues which starts from patient needs and ends with transfusion of the needed components to patients (RBC).

* Corresponding author. Tel.: +98 21 88021067; fax: +98 2188013102

E-mail: mrabani@ut.ac.ir (M. Rabbani)

(C) 2017 Growing Science Ltd. All rights reserved.

doi: $10.5267 /$ j.ijiec.2016.7.005 
Blood cannot be produced in laboratories and the only source of blood supply is blood donation which makes the blood supply very problematic and critical. Blood supply chain involves different components including patients, hospitals or other health providers, blood centers (BCs), blood mobiles, and donors. Each component has different concerns and priorities which should be considered in blood supply chain to fully manage collection, distribution, and storage operations as well as scarcity and wastage issues. Study on blood supply chain started in the late 60 s and still it is in significance. Wide varieties of problems, challenges, and case studies have been addressed during these years. However, due to the complexity of human behavior and environments dealt with different tiers of blood supply chain, applied solution methods, approaches, and performance measures should be improved.

Beliën and Forcé (2012) presented a comprehensive literature review on inventory and supply chain management of blood products. They have classified related papers based on different types of blood products and different approaches and solution methods. They stated that study on blood supply chain peaked in the 80s and dropped till the turn of the century. Recently, due to growing challenges in blood supply chain management, both practitioners and academicians have highlighted the criticality of studying blood supply issues. According to American Red Cross, total collection of different blood components was 17.3 million units in 2011 which shows a significant decline compared to 2008 (about 9.1 percent). While almost $10.3 \%$ of hospitals reported at least one day on which non-surgical blood need could not be fulfilled. Moreover, the total number of reported "days incomplete orders" was 37857 unweighted days in 2011(US Department of Health and Human Services 2011). Although the survey shows distinguished improvement compared to 2008, the results are not satisfactory, since the impact of unmet demand can result in serious health problems or even death. Recently, Osorio et al. (2015) presented a structured review on quantitative models of blood supply chain. They have cited different approaches and models in each echelon of blood supply chain and identified modeling approaches, decisions and characteristics which have been rarely studied.

Collection stage of blood supply chain has been widely studied. Main decisions in this stage are location of donor blood drawing facilities and corresponding capacity decisions, collection methods, donor management, routing and transportation fleet decisions (Osorio et al. 2015). To this end, several methods have been introduced and applied in the literature to improve recruitment and retention practices. One of the early reviews on blood donor management was provided by Oswalt and Robert (1977) in which blood donor motivations, characteristics, and personalities were thoroughly discussed. Many studies in this regard have been addressed tools and methods to find critical factors affecting blood donation and easing donor segmentation. Veldhuizen et al. (2009) studied impact of demographic factors on resigning donation and being a multi gallon donor to help donor management process. Veldhuizen et al. (2013) presented a novel tool for donor segmentation in order to increase donor population in blood supply chain. They stated that for donor cycle and related tools, it is key a factor to clearly make a distinction between active donors and inactive ones. Moreover, stopped donors should be listed separately to analyze causes. In a recent paper, Dongen et al. (2014) applied logistic regression technique on data gathered from donors who had donated blood for the first time to identify influencing measures on donor retention.

The characteristics of collection sites have been studied by Şahinyazan et al. (2015). Several strategies for modifying set up, staff allocation, and work rules have been tested on Red Cross bloodmobiles to flatten out excessive waiting lines and blood donation time. A Markovian population model was applied by Cumming et al. (1976) to assist regional blood supplier lighten the imbalances of blood supply and demand. The proposed model was one of the early planning models for blood collection. Magali et al. (2011) studied interactions between platelet supply chain processes of the Stanford University Medical Center Transfusion Service and the Stanford Blood Center. The analyses resulted in lower platelet outdate. Ghandforoush et al. (2010) also addressed platelet supply chain problem. They proposed an integer model for scheduling of whole blood transportation from bloodmobiles to blood center intended for platelet separation purposes. They presented a prototype decision system based on the model. In a study, Madden et al. (2007) modeled the procurement of red blood cells with both double red blood cell and whole blood collection using a cohort simulation. Katsaliaki (2008) conducted a specific analysis using 
data from National Blood Service and developed a simulation model of UK supply chain of blood products (red blood cells and blood platelet) to determine those policies which are more practical for better cost effective management of blood supply chains. Zahraee et al. (2015) proposed a computer simulation model to increase blood supply chain efficiency of Finish healthcare system. Haijema et al. (2007) proposed a combined Markov dynamic programming and simulation approach for production management and inventory optimization of blood platelet and applied it to a Dutch blood bank. Haijema et al. (2009) also studied the production and inventory management of blood platelets in breaks such as Easter and Christmas by applying stochastic dynamic programming and simulation. Van Dijk et al. (2009) combined stochastic dynamic programming and simulation to optimize blood platelet production. Nagurney et al. (2012) developed an optimization model for procurement, testing, processing, and distribution of blood using variational inequality approach for model formulation and solution. Hemmelmayr et al. (2010) developed a mathematical model for blood supply chain. They presented an integer programming model and variable neighborhood search to plan delivery routes for blood products from blood center(s) to hospitals. The model accounts for natural uncertainty of blood product usage in hospitals.

The rest of paper is organized as follows: Section 2 describes the problem and the mathematical formulation is presented in this Section. Methodology is discussed in Section 3. Some experiments are conducted and numerical results are shown in section 4. Finally, conclusion remarks and future research are provided in Section 5.

\section{Problem Description}

Platelets are regarded as the most perishable blood products which deteriorate between 5 to 7 days after production. There are two ways to derive platelets; the first way is using a centrifuge to separate the platelet rich plasma from whole blood called random platelet and the second way is to use apheresis tool. In case of random platelet production, whole blood of donated should be transported periodically due to 6 hours limit on separation from red blood cell (Ghandforoush et al., 2010). The aforementioned characteristics of platelets have intensified the existing complexities of blood supply chain. One of the critical decisions in platelet supply chain is to determine the location of bloodmobiles and corresponding tour for periodic shuttling of shuttles while maximizing the amount of collected blood and minimizing total cost (Fontaine et al., 2009). The aforementioned problem is addressed in this paper.

\subsection{Model Development}

Let $G=(N, E)$ be a geographical network where $N \backslash\{1\}$ is the set of potential stops for the bloodmobiles and node $\{1\}$ is the given location of blood center (BC). Set of edges $E$ represents the roads among these nodes. A cost $c_{i j}$ and a time $t_{i j}$ which may include service time is associated with each edge $(i, j)$ where $i \neq j$. The blood potentials of node is assumed to be a fuzzy number. For each bloodmobile $i$ a time window $\left[a_{i}, b_{i}\right]$ is defined and the shuttles must arrive at the bloodmobile before $b_{i}$. If it arrives before the time window opens, it has to wait to collect platelets or the whole blood. To address the problem, two models are developed. In the first model, blood collection sites for each day $(d)$ are identified and in the second model the tours for periodic shuttling are specified. The following notations are used:

$$
\begin{aligned}
X_{i j k} & =\left\{\begin{array}{l}
1, \text { If shuttle } k \text { travels directly from bloodmobile } i \text { to bloodmobile } j \\
0, \text { Otherwise }
\end{array}\right. \\
Y_{i j d} & = \begin{cases}1, & \text { If a bloodmobile travels from node } i \text { to node } j \\
0, & \text { Otherwise }\end{cases}
\end{aligned}
$$


$r_{i k}= \begin{cases}1, & \text { If vehicle } k \text { visits node } i \\ 0, & \text { Otherwise }\end{cases}$

$\mathrm{Cap}_{k} \quad$ The capacity of vehicle $k$

$c_{i j} \quad$ The arc length between node $i$ and node $j$

$\mathrm{c}_{i j k}^{\prime} \quad$ The cost of using vehicle $k$ for edge $(i, j)$

$s_{i k} \quad$ The time vehicle $k$ starts servicing bloodmobile $i$.

$m \quad$ Number of bloodmobiles

$\tilde{w}_{j}=\left(w_{j}^{p}, w_{j}^{m}, w_{j}^{o}\right) \quad$ Fuzzy number for blood potential at node $j$

The proposed model is as follows:

$$
\begin{aligned}
& \min \sum_{i \in N, j \in N, k \in V}\left(c_{i j}+\mathrm{c}_{i j k}^{\prime}\right) x_{i j k}+c_{i j} Y_{i j d} \\
& \max \sum_{i \in N, j \in \mathbb{N}} \tilde{w}_{j} Y_{i j d} \\
& \sum_{i \in N} Y_{i j d}=\sum_{f \in N} Y_{i f(d+1)} \quad \forall j \in\{2, \ldots, N\}, \forall d \in\{1, \ldots, d-1\} \\
& \sum_{j \in N} Y_{1 j 1}=m \\
& \sum_{i \in\{2, \ldots, N\}, j \in N} Y_{i j 1}=0 \\
& \sum_{j \in N, d \in D} Y_{1 j d}=m \\
& \sum_{j \in N, d \in D} Y_{j l d}=m \\
& \tilde{w}_{j} Y_{i j d} \leq C a p_{k} \quad \forall i, j, k, d \\
& Y_{i j d} \in\{0,1\} \quad \forall i, j \in N, \forall d \in D
\end{aligned}
$$

Objective function (1) (TC) minimizes cost of traveling for both bloodmobiles and shuttles and the second objective, Potential Blood Collection, maximizes the amount of collected blood. Constraints (3) specifies the flow balance conditions such that if there is a bloodmobile coming to node $j$ on day $d$ there should be also an outgoing one from node $j$ on day $d+1$. Constraint (4) forces all bloodmobile tours to start on the first day. Since, the objective is to maximize collected blood, the vehicles should never remain idle when there is available blood to be collected. Thus, in the current setting this constraint serves as a valid inequality. Constraint (5) presents tours starting from any node other than the depot. Constraint (6) restricts the number of vehicles leaving the depot. Constraint (7) ensures all bloodmobiles return to the depot. Eqs. (8) are associated with vehicles capacity constraints. Finally, Eq. (9) is the integrality constraints.

Model 2:

$\min z_{2}=D \sum_{k \in V} \sum_{j \in N^{\prime}} x_{0 j k}+\sum_{k \in V} \sum_{i \in N^{\prime}} \sum_{j \in N^{\prime}} d_{i j} x_{i j k}$

Subject to

$$
\begin{aligned}
& \sum_{k \in V} r_{i k}=1 \quad \forall i \in N^{\prime}, \\
& \sum_{i \in N^{\prime}} w_{i} r_{i k} \leq c a p \quad \forall k \in V, \\
& \sum_{j \in N^{\prime}} x_{0 j k}=1 \quad \forall k \in V, \\
& \sum_{i \in N^{\prime}} X_{i h k}-\sum_{j \in N^{\prime}} X_{h j k}=0 \quad \forall h \in N^{\prime}, \forall k \in V \\
& \sum_{j \in N^{\prime}} x_{i j k} \geq r_{i k} \quad \forall i \in N^{\prime}, \forall k \in V, \\
& X_{i j k}\left(s_{i k}+t_{i j}-s_{j k}\right) \leq 0 \quad \forall i, j \in N^{\prime}, \forall k \in V
\end{aligned}
$$




$$
\begin{aligned}
& X_{i 0 k}\left(s_{i k}+t_{i 0}-b_{0}\right) \leq 0 \quad \forall i \in N^{\prime}, \forall k \in V \\
& a_{i} \leq s_{i k} \leq b_{i} \quad \forall i \in N^{\prime}, \forall k \in V, \\
& S_{0 k}=a_{0} \quad \forall k \in V, \\
& r_{i k} \in\{0,1\} \quad \forall i \in N^{\prime}, \forall k \in V, \\
& x_{i i k}=0 \quad \forall \mathrm{i} \in \mathrm{N}^{\prime}, \forall k \in V, \\
& x_{i j k} \in\{0,1\} \quad \forall i, j \in N^{\prime}, \forall k \in V .
\end{aligned}
$$

The objective function (10) minimizes the total travel cost. The constraints (11) ensure that each customer is visited exactly once, and constraints (12) state that a vehicle can only be loaded up to its capacity. Eqs. (13-15) indicate that each vehicle must leave depot 1; after a vehicle arrives at a bloodmobile it has to leave for another destination; and finally, all vehicles must arrive at the BC. The inequalities (16) and (17) establish the relationship between the vehicle departure time from a bloodmobile and its immediate successor. Finally constraints (18) and (19) affirm that the time windows are observed. Constraints (20)(22) are the integrality constraints.

\section{The proposed solution approach}

Model 1 is a multi-objective possibilistic integer linear programming model. Solving this problem needs a two phase approach; in the first phase, the original model is converted into an auxiliary crisp multi objective integer linear model and in the second phase, a fuzzy programming solution is applied to find preferred compromise solution for each of the crisp models. The calculated results from solving model 1 are incorporated into the second model. Model 2 is solved via s simulated annealing (SA) meta heuristics approach.

\subsection{Equivalent auxiliary crisp model for Model1}

There are several approaches for converting fuzzy models to auxiliary crisp one (Jiménez et al., 2005; Wang, R. C., \& Liang). Here, the expected value operator and chance constrained operator based on Me measure introduced by $\mathrm{Xu}$ and Zhou (2013) is applied for defuzzification of the model.

Consider the following general multi objective model:

$$
\begin{aligned}
& \max \left[f_{1}(x, \varsigma), f_{2}(x, \varsigma), \ldots, f_{m}(x, \varsigma)\right] \\
& \text { s.t. }\left\{\begin{array}{l}
g_{n}(x, \zeta) \leq 0 ; n=1,2, \ldots, \mathrm{N} \\
x \in X
\end{array}\right.
\end{aligned}
$$

The following multi objective model with expected objectives and chance constraints is acquired (ECM):

$$
\begin{aligned}
& \max \left[E\left[f_{1}(x, \varsigma)\right], \mathrm{E}\left[f_{2}(x, \varsigma)\right], \ldots, \mathrm{E}\left[f_{m}(x, \varsigma)\right]\right] \\
& \text { s.t. }\left\{\begin{array}{l}
\operatorname{ch}\left\{g_{n}(x, \zeta) \leq 0\right\} \geq \tau_{n} ; n=1,2, \ldots, \mathrm{N} \\
x \in X
\end{array}\right.
\end{aligned}
$$

where:

$$
\mathrm{E}[\varsigma]=\int_{0}^{+\infty} M e\{\zeta \geq n\} d n-\int_{-\infty}^{0} M e\{\zeta \leq n\} d n
$$

Provided that at least one of the integrals is finite. Based on Xu and Zhou (2013) for $E[\varsigma]$ triangular fuzzy variable $\varsigma=\left(n_{1}, n_{2}, n_{3}\right)$ is: 


$$
\mathrm{E}[\varsigma]= \begin{cases}\frac{\lambda}{2} n_{1}+\frac{1}{2} n_{2}+\frac{(1-\lambda)}{2} n_{3} & \text { if } n_{3} \leq 0 \\ \frac{\lambda}{2}\left(n_{1}+n_{2}\right)+\frac{\lambda n_{3}^{2}-(1-\lambda) n_{2}^{2}}{2\left(n_{3}-n_{2}\right)} & \text { if } n_{2} \leq 0 \leq n_{3} \\ \frac{(1-\lambda) n_{2}^{2}-\lambda n_{1}^{2}}{2\left(n_{2}-n_{1}\right)}+\frac{\lambda}{2}\left(n_{2}+n_{3}\right) & \text { if } n_{1} \leq 0 \leq n_{2} \\ \frac{(1-\lambda)}{2} n_{1}+\frac{1}{2} n_{2}+\frac{\lambda}{2} n_{3} & \text { if } 0 \leq n_{1}\end{cases}
$$

where $\lambda$ is optimistic pessimistic parameter. In order to measure the chance of a fuzzy event, the $M e$ measure is used as follows:

$$
\operatorname{ch}\left\{g_{n}(x, \zeta) \leq 0\right\} \geq \tau_{n} \Leftrightarrow \operatorname{Me}\left\{g_{n}(x, \zeta) \leq 0\right\} \geq \tau_{n}
$$

where $\tau_{n}, n=1,2, \ldots, N$ are the decision maker's confidence levels and $\operatorname{Me}\{A\}=\operatorname{Nec}\{A\}+\lambda(\operatorname{Pos}\{A\}-\operatorname{Nec}\{A\})$. When $\lambda=1, \operatorname{Me}\{A\}=\operatorname{Pos}\{A\}$ which shows that the decision maker is optimistic and it is the maximal chance that $A$ holds. When $\lambda=0, \operatorname{Me}\{A\}=\operatorname{Nec}\{A\}$ which shows that the decision maker is pessimistic and it is the minimal chance that $A$ holds. Therefore, Eq. (24) can be transformed into lower approximation model (LAM) and upper approximation model (UAM) which are as follows respectively:

$$
\begin{aligned}
& \max \left[E\left[f_{1}(x, \varsigma)\right], \mathrm{E}\left[f_{2}(x, \varsigma)\right], \ldots, \mathrm{E}\left[f_{m}(x, \varsigma)\right]\right] \\
& \text { s.t. }\left\{\begin{array}{l}
\operatorname{Nec}\left\{g_{n}(x, \zeta) \leq 0\right\} \geq \tau_{n} n=1,2, \ldots, \mathrm{N} \\
x \in X
\end{array}\right. \\
& \max \left[E\left[f_{1}(x, \varsigma)\right], \mathrm{E}\left[f_{2}(x, \varsigma)\right], \ldots, \mathrm{E}\left[f_{m}(x, \varsigma)\right]\right] \\
& \text { s.t. }\left\{\begin{array}{l}
\operatorname{Pos}\left\{g_{n}(x, \zeta) \leq 0\right\} \geq \tau_{n} n=1,2, \ldots, \mathrm{N} \\
x \in X
\end{array}\right.
\end{aligned}
$$

According to above description, in comparison with model 1, Eq. (2) and Eq. (8) must be changed in order to obtain the equivalent lower approximation and upper approximation models. Hence, Eq. (2) will be converted to the expected objective as follows:

$$
\max \mathrm{E}[O b j 2]=\sum_{\mathrm{i}, j} Y_{i j d}\left[\left(\frac{1-\lambda}{2}\left(w_{j}-\alpha_{j}^{w}\right)+\frac{w_{j}}{2}+\frac{\lambda}{2}\left(w_{j}+\beta_{j}^{w}\right)\right)\right]
$$

Eq. (30) holds for both LAM and UAM models. Also, Eq. (8) will be converted to the following constraints for LAM and UAM models respectively:

$$
\begin{aligned}
& Y_{i j d} \leq\left(1 / w_{j}-\tau_{1} \alpha_{j}^{w r}\right) \quad \forall i, j, d \\
& Y_{i j d} \leq\left(1 / w_{j}+\left(1-\tau_{1}\right) \beta_{j}^{w}\right) \quad \forall i, j, d
\end{aligned}
$$

It should be noticed that the remaining equations will be kept unchanged and are the same for both LAM and UAM models.

\subsection{The proposed fuzzy solution approach}

To solve multi objective linear programming models, several methods have been proposed in the literature. Among them fuzzy approaches have been mostly applied in recent years since they help 
decision maker choose efficient solution based on the satisfaction degree and relative importance of each objective (Selim \& Ozkarahan, 2008). There are many fuzzy programming solution approaches (Luhandjula, 2015; Torabi \& Hassini, 2008), among them the TH method which yields efficient solutions is applied in this paper. To solve LAM and UAM models derived from the previous step by TH method, the subsequent steps should be followed (Torabi \& Hassini, 2008; Pishvaee \& Torabi, 2010):

Step 1: Determine the positive ideal solution $\left(Z O_{\eta}^{P I S}, \omega_{\eta}^{P I S}\right)$ and negative ideal solution $\left(Z O_{\eta}^{N I S}, \omega_{\eta}^{N I S}\right)$ for each objective function based on the given $\tau_{n}$ and $\lambda$ for both LAM and UAM models. The positive ideal solution can be obtained by solving aforementioned models for each objective function separately and the negative ideal solution can be estimated as follows:

$$
Z O_{\eta}^{N I S}=\max _{k=T C, A M S, R I S K}\left[Z O_{\eta}^{P I S}\left(\omega_{k}^{P I S}\right)\right] ; \quad \eta=T C, R I S K
$$

and

$$
Z O_{\eta}^{N I S}=\max _{k=T C, A M S, R I S K}\left[Z O_{\eta}^{P I S}\left(\omega_{k}^{P I S}\right)\right] ; \quad \eta=A M S
$$

where $\omega_{\eta}^{P I S}$ is decision vector associated with the PIS of $\eta$ th objective function.

Step 2: Determine a linear membership function for each objective function:

$$
\begin{aligned}
& \mu_{o b j 1}(x)= \begin{cases}1 & \text { if } Z O_{o b j 1}<Z O_{o b j 1}^{\beta-P I S} \\
\frac{Z O_{o b j 1}^{\beta-N I S}-Z O_{o b j 1}}{Z O_{o b j 1}^{\beta-N I S}-Z O_{o b j 1}^{\beta-P I S}} & \text { if } Z O_{o b j 1}^{\beta-P I S} \leq Z O_{o b j 1} \leq Z O_{o b j 1}^{\beta-N I S} \\
0 & \text { if } Z O_{o b j 1}>Z O_{o b j 1}^{\beta-N I S}\end{cases} \\
& \mu_{o b j 2}(x)= \begin{cases}1 & \text { if } Z O_{o b j 2}>Z O_{o b j 2}^{\beta-P I S} \\
\frac{Z O_{o b j 2}-Z O_{o b j 2}^{\beta-N I S}}{Z O_{o b j 2}^{\beta-P I S}-Z O_{o b j 2}^{\beta-N I S}} & \text { if } Z O_{o b j 2}^{\beta-N I S} \leq Z O_{o b j 2} \leq Z O_{o b j 2}^{\beta-P I S} \\
0 & \text { if } Z O_{o b j 2}<Z O_{o b j 2}^{\beta-N I S}\end{cases}
\end{aligned}
$$

Step 3: convert LAM and UAM models into a corresponding equivalent single objective ILP as below:

$$
\begin{array}{ll}
\max \sigma(\omega) & =\psi \sigma_{0}+(1-\psi) \sum_{\eta} \theta_{\eta} \mu_{\eta}(\omega) \\
\text { st } ; & \sigma_{0} \leq \mu_{\eta}(\omega) \\
& \omega \in F(\omega) ; \psi, \sigma_{0} \in[0,1]
\end{array}
$$

where $\theta_{\eta}$ and $\psi$ are relative importance of the $\eta^{\text {th }}$ objective and coefficient of compensation, respectively. $\theta_{\eta}$ can be obtained either by asking directly from decision makers or through MCDM techniques. $F(\omega)$ indicates the constraints of LAM model or UAM.

\subsection{Simulated Annealing Approach}

SA (Brooks et al., 1995) is one of the most popular metaheuristics that have been applied broadly to solve many combinatorial optimization problems (Rabbani et al., 2016). In fact, it is an iterative method 
that uses random local search technique based on the principles of Metallurgy. In this method, an initial solution is first generated, randomly. Then a random neighbor solution of initial solution is generated using a small change in one or a combination of some variables. In SA, the neighboring solution is generated randomly. If the candidate solution is better than the current solution, a move to the candidate solution is made, but if the candidate does not improve the current solution, there is still a chance of transition based on "Boltzmann" distribution probability. In this paper, the initial temperature $(T)$, cooling rate $(\alpha)$, Boltzman constant $(K)$, number of outer loop and finally the number of inner loop are set as 20 , $0.99,4,2000$ and 500, respectively. Interested readers for more detail about SA can refer to Lenstra (1997) and Pirlot (1992).

\subsection{Solution Representation}

In order to apply SA for solving the problem which associates with the vehicle routing problem, solution representations for vehicle routes should be specified. Vehicles start from the RBC, and then visit the blood collection sites based on the associated orders in solution representation. Therefore, a vehicle should be arrived within the blood collection sites' time window. Vehicles which arrive before starting time need to wait until the allowable time of delivery. Since there are multiple vehicles and each vehicle has a specific route, the RBC is copied in solution representation as route separator. In this paper, a $|1| \times|\mathrm{I}+\mathrm{J}-1|$ binary matrix is used for solution representation in which $I$ is the number of collection sites and $J$ is the number of vehicles. RBCs are the strings whose values are bigger than $I$ (and lower than $I+J)$. For instance, Fig. 1 shows a solution representation for 3 vehicles and 9 blood collection sites. The first route is 3,5 , and 7; the second route is 1,6,2, and 9; and the last route which is for vehicle 3 is 8 and 4. It should be noted that each route begin from the RBC, and values 10 and 11 are associated with the $\mathrm{RBC}$ and act as a separator between the routes.

\begin{tabular}{|l|l|l|l|l|l|l|l|l|l|l|}
\hline 3 & 5 & 7 & 10 & 1 & 6 & 2 & 9 & 11 & 8 & 4 \\
\hline
\end{tabular}

Fig. 1. SA solution representation

The above thee routes can be shown graphically as Fig. 2. As the problem is a closed VRP, each routes starts from the RBC and ends at the RBC.

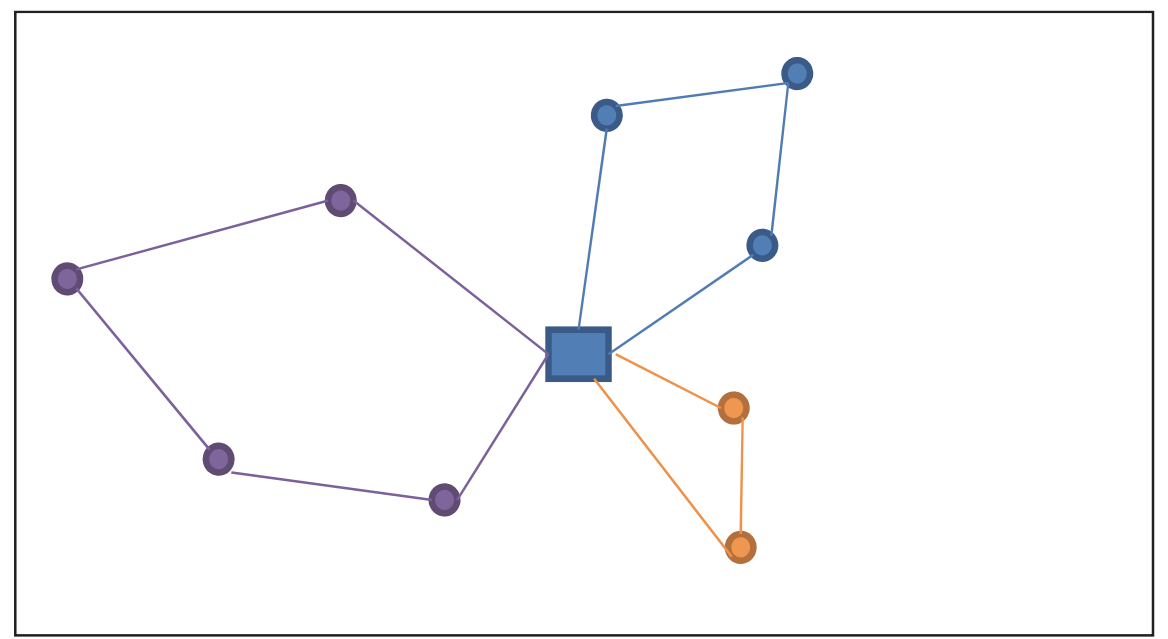

Fig. 2. A graphical solution corresponding to the solution representation

In a repetition of the proposed SA procedure, a new state of solution is generated from the present state by one to one transformation rule for generating neighbors. According to this rule, a number is exchanged with another one in the string. An example is illustrated in Fig. 3. 


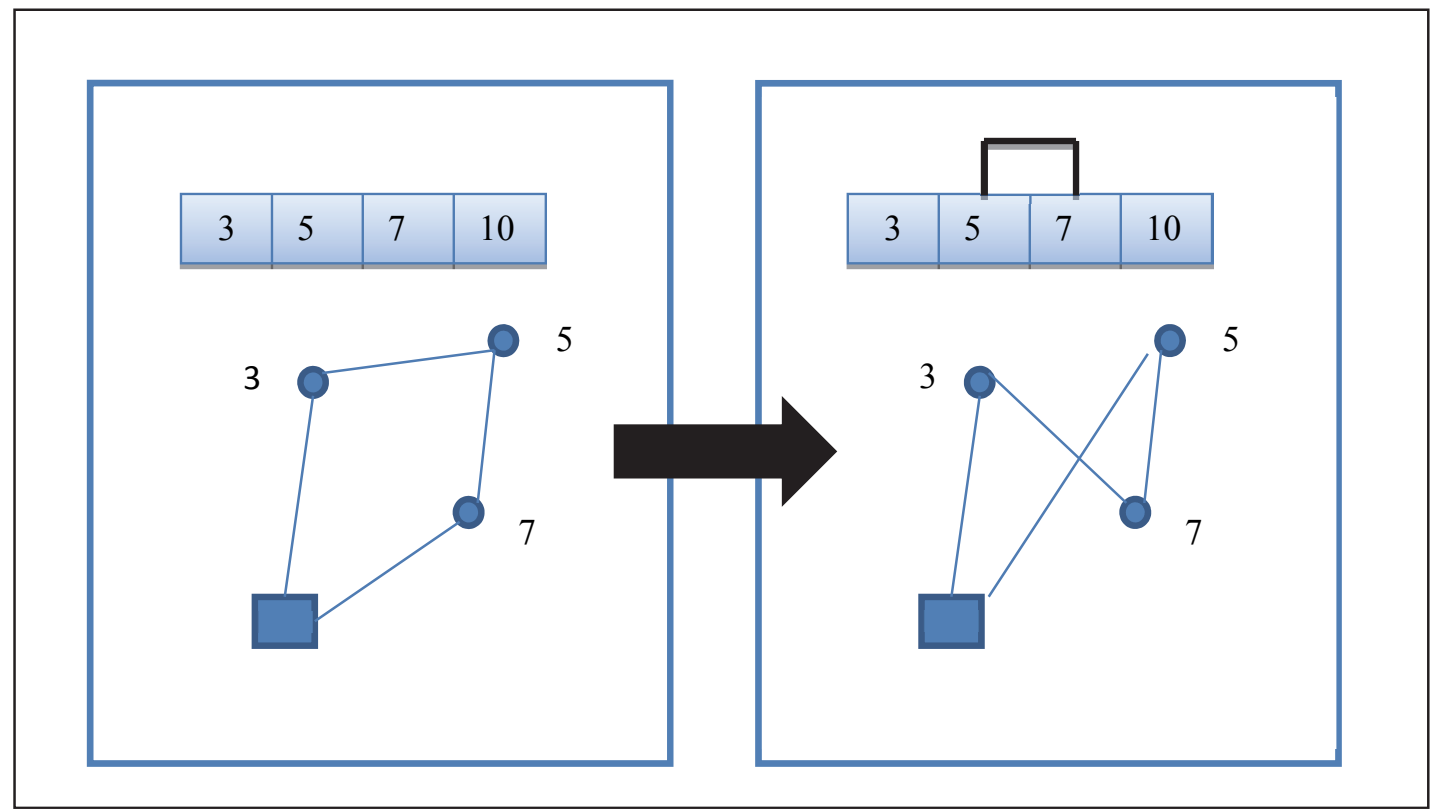

Fig. 3. Transformation rule for generating neighbors

\section{Computational results}

To demonstrate the capability of the proposed models, several problems have been solved and the results are stated and analyzed due to random generated distribution functions samples which is given in Table 1. It should be noted that all the problems in model 1 were coded in GAMS and solved by CPLEX 12 solver with Core-i5, 2.5GHz of CPU and 4GB of RAM processor. In addition, all the problems in model 2 were coded in Matlab and solved with Core-i5, 2.5GHz of CPU and 4GB of RAM processor.

\subsection{Sensitivity Analysis}

In this section, an example consists of 15 bloodmobiles (collection sites), one blood center, and 4 vehicles which collect the potential blood in bloodmobile zones and deliver to the blood center is presented. Data for most of the parameters are generated randomly. For fuzzy parameters the most possible value $\left(n^{m}\right)$ is generated based on the corresponding distribution and the most pessimistic and optimistic values are calculated as follows: $n^{p}=0.8 n^{m} ; n^{o}=1.2 n^{m}$. The remainder data for the illustrative example is given in Table 1. Moreover the relative importance of each objective $\theta_{\eta}$ is provided by the decision maker as $\left(\theta_{1}=0.8, \theta_{2}=0.2\right)$ which shows greater importance of the first objective function over the second one.

Table 1

Data for illustrative example

\begin{tabular}{cc}
\hline Parameter & Data generation range \\
\hline$c a p_{k}$ & $U(300,2000)$ \\
$d_{i, j}$ & $U(100,300)$ \\
$w_{j}^{m}$ & $N(100,800)$ \\
\hline
\end{tabular}

Using TH approach, an equivalent single objective model contains different parameters and different DMs may take different attitude towards each of the parameters. It has made us to perform sensitivity analysis over some of the main model parameters. In the first part, a sensitivity analysis is conducted on optimistic-pessimistic parameter $(\lambda)$ and confidence level parameter $\left(\tau_{n}\right)$ based on the given coefficient of compensation parameter $(\psi)$. Since the first objective is more important than the second one as stated 
by $\left(\theta_{l}=0.8, \theta_{2}=0.2\right)$, unbalanced solution with higher degree of satisfaction for the first objective is more attractive. Consequently, $\psi$ is set to 0.4 in this part and also, it is assumed that all $\tau_{n}$ have the same value.

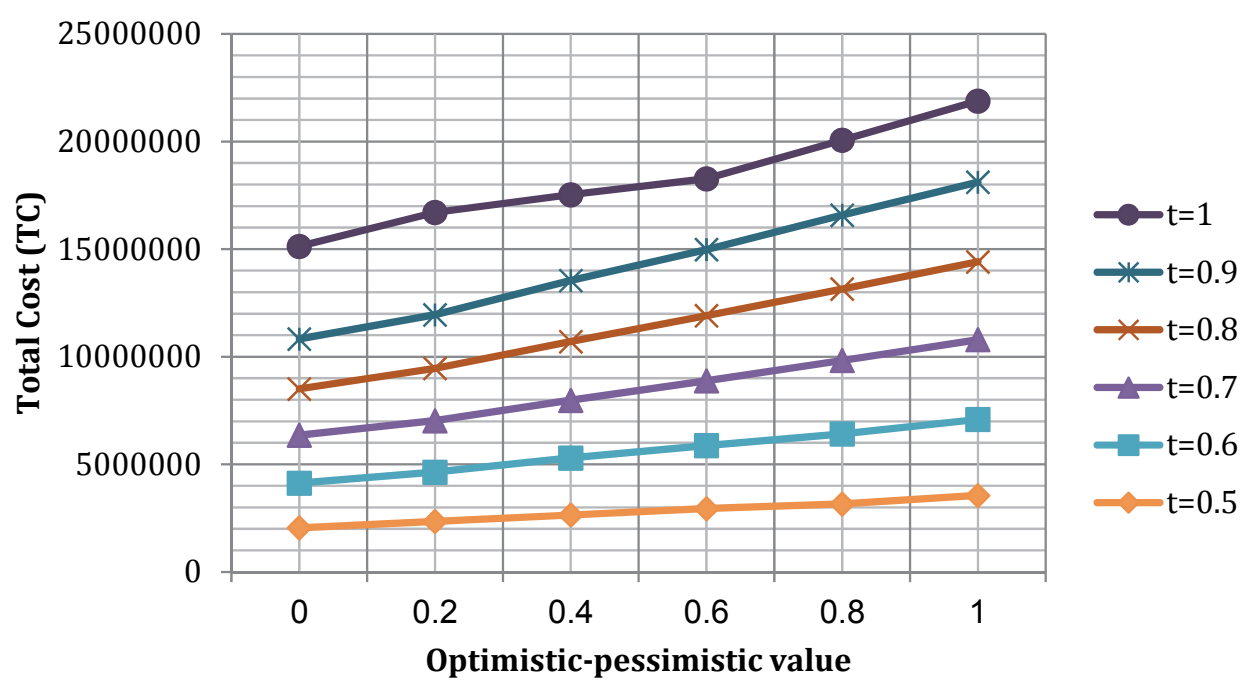

Fig. 1. Total costs based on the different optimistic-pessimistic values and confidence levels for UAM corresponding equivalent single objective ILP

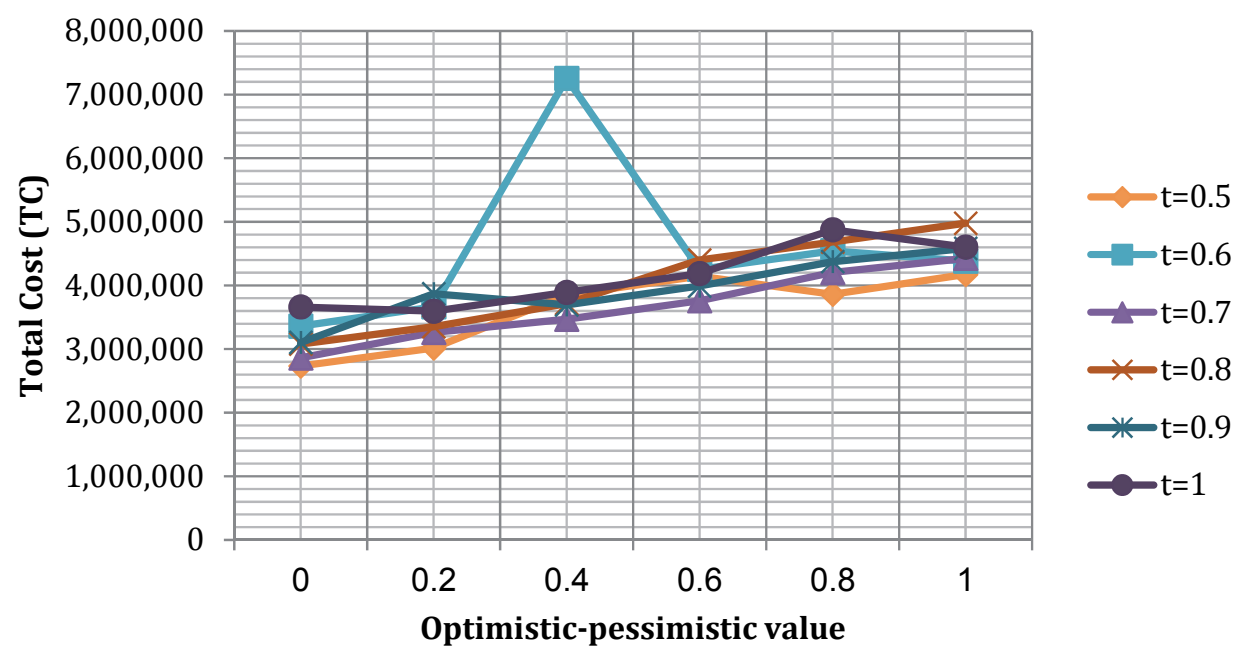

Fig. 2. Total costs based on the different optimistic-pessimistic values and confidence levels for LAM corresponding equivalent single objective ILP

Fig. 3 and Fig. 4 show the effect of parameters $\lambda$ and $\tau_{n}$ on the first objective (TC) of corresponding equivalent single objective ILP $(\sigma(\omega))$ for both LAM and UAM. It is rather obvious that under the same confidence levels, as the optimistic-pessimistic parameter increases, the value of TC increases too. In fact, for the cost objective, $\lambda$ is a pessimistic parameter. When the decision makers have more pessimistic attitudes toward the fuzzy parameters, they would set higher value to $\lambda$ and then the cost objective would have higher value. Furthermore, with the same optimistic-pessimistic parameter, when the confidence level increases, the value of cost objective increases. When the confidence level increases, the feasible region shrinks so the derived solution is expected to be worse. Noteworthy, with the same parameters value of cost function for LAM is higher than the UAM because the feasible region in UAM is greater than LAM. Similar to the cost objective, as the confidence level increases under the same $\lambda$, the value of 
AMS gets worse. Notably, parameter $\lambda$ does not significantly affect AMS value, owing to the fact that it is a deterministic objective (Fig. 5 and Fig. 6). The satisfaction degree of each objective for LAM and UAM corresponding equivalent single objective for different values of parameters $\lambda$ and $\tau$ are shown in Table 2.

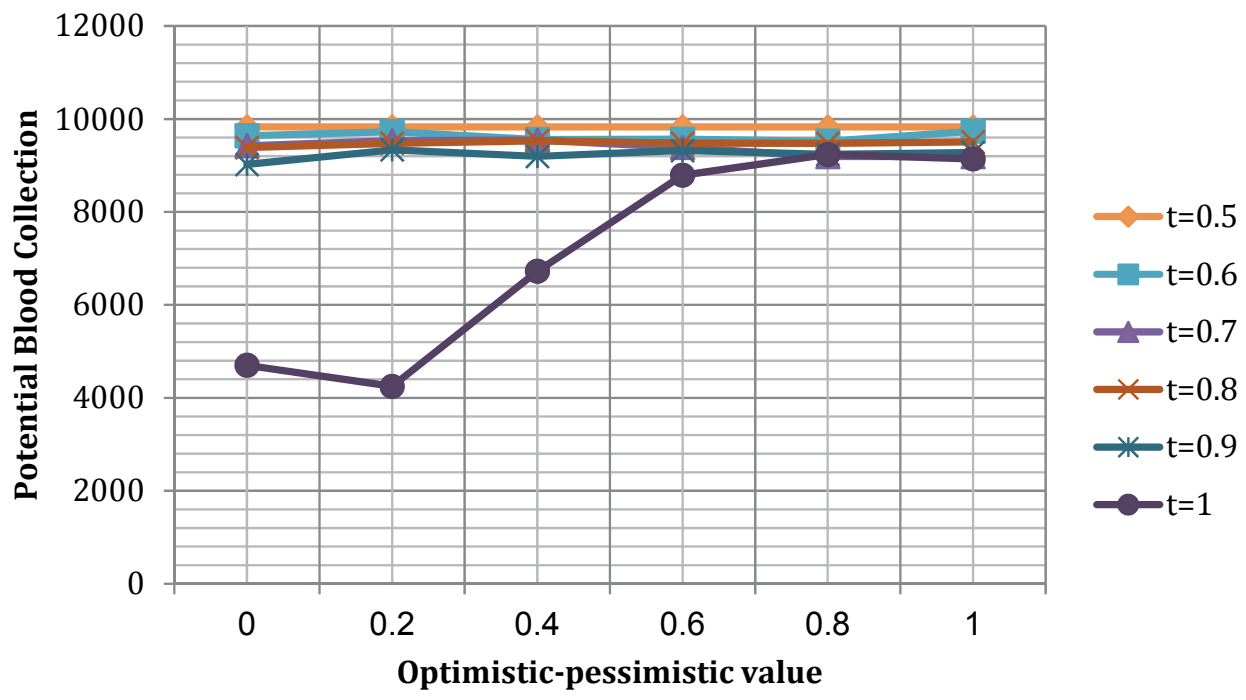

Fig. 3. Potential blood collection based on the different optimistic-pessimistic values and confidence levels for UAM corresponding equivalent single objective ILP

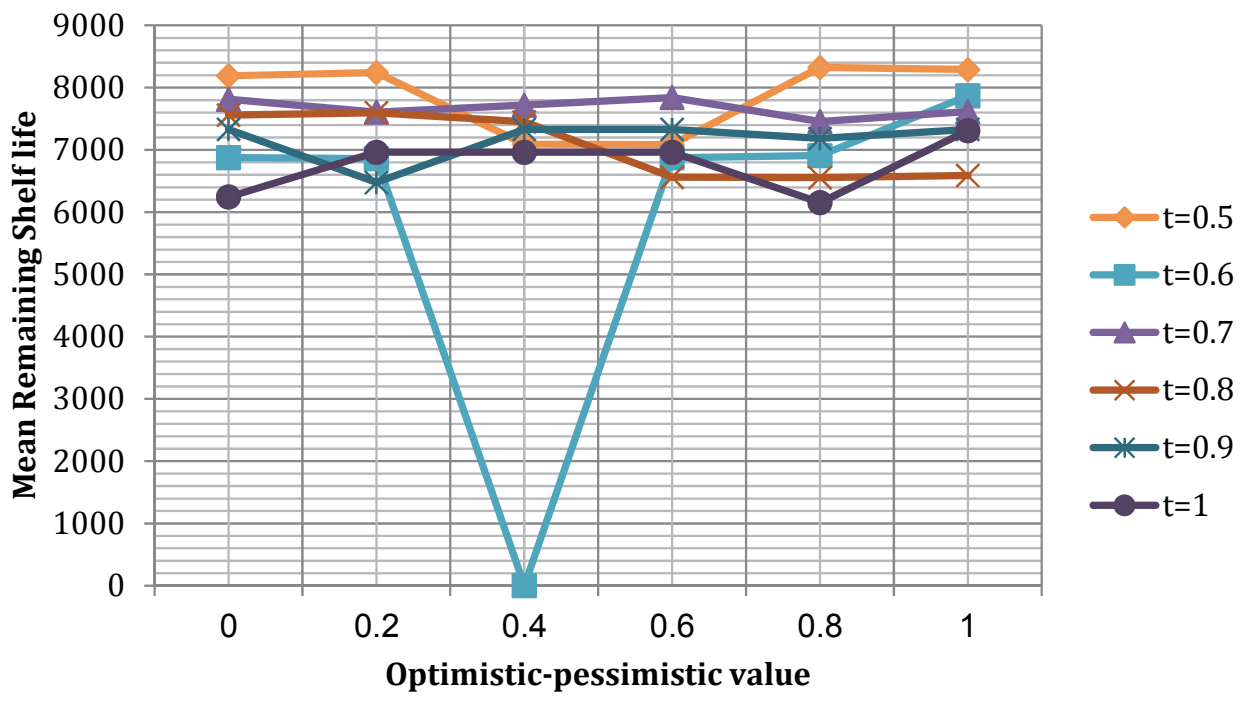

Fig. 4. Potential blood collection based on the different optimistic-pessimistic values and confidence levels for LAM corresponding equivalent single objective ILP

In the second part of sensitivity analysis, the LAM and UAM parameters are set to $\lambda=0.6 ; \tau=0.8$ and then different satisfaction degrees of objectives based on the different values of coefficients of compensations as well as the weight values for the objective functions (i.e., $\theta_{n}$ ) are calculated and the results are shown in Table 3. The results presented in Table 3 show that as the coefficients of compensation and the importance of the first objective increase, the value of both objective functions rises. For the second model, different number of vehicles and bloodmobiles are considered based on the results obtained from model 1 given in Table 4. The graphical representation of problem instances 5 and 9 are presented in Fig. 7 and Fig. 8, respectively. 
Table 2

The satisfaction degree of each objective for LAM and UAM Corresponding equivalent single objective for different values of parameters $\lambda$ and $\tau$

\begin{tabular}{lccc}
\hline & $\lambda=0$ & $\lambda=0.2$ & $\lambda=0.4$ \\
\hline$\tau=0.5$ & $\{(0.985,1.000),(0.984,1.000)\}$ & $\{(0.985,1.000),(0.986,1.000)\}$ & $\{(0.985,1.000),(0.898,0.999)\}$ \\
$\tau=0.6$ & $\{(0.984,1.000),(0.898,0.999)\}$ & $\{(0.996,1.000),(0.897,0.999)\}$ & $\{(0.985,1.000),(0.959,0.996)\}$ \\
$\tau=0.7$ & $\{(0.973,1.000),(0.993,1.000)\}$ & $\{(0.994,1.000),(0.973,1.000)\}$ & $\{(0.994,1.000),(0.988,1.000)\}$ \\
$\tau=0.8$ & $\{(0.998,1.000),(0.974,1.000)\}$ & $\{(1.000,1.000),(0.978,1.000)\}$ & $\{(1.000,1.000),(0.996,1.000)\}$ \\
$\tau=0.9$ & $\{(0.978,1.000),(0.979,1.000)\}$ & $\{(0.997,1.000),(0.887,0.999)\}$ & $\{(0.991,1.000),(0.979,1.000)\}$ \\
$\tau=1$ & $\{(0.687,0.998),(0.897,0.999)\}$ & $\{(0.663,0.997),(0.962,1.000)\}$ & $\{(0.829,0.999),(0.962,1.000)\}$ \\
\hline \multicolumn{2}{c}{$\lambda=0.6$} & $\lambda=0.8$ & $\lambda=1$ \\
\hline$\tau=0.5$ & $\{(0.985,1.000),(0.898,0.999)$ & $\{(0.994,1.000),(0.993,1.000)\}$ & $\{(0.980,1.000),(0.991,1.000)\}$ \\
$\tau=0.6$ & $\{(0.993,1.000),(0.897,0.999)\}$ & $\{(0.997,1.000),(0.899,0.999)\}$ & $\{(0.999,1.000),(0.975,1.000)\}$ \\
$\tau=0.7$ & $\{(0.990,1.000),(0.989,1.000)\}$ & $\{(0.974,1.000),(0.964,1.000)\}$ & $\{(0.974,1.000),(0.976,1.000)\}$ \\
$\tau=0.8$ & $\{(1.000,1.000),(0.897,0.999)\}$ & $\{(1.000,1.000),(0.897,0.999)\}$ & $\{(1.000,1.000),(0.897,0.999)\}$ \\
$\tau=0.9$ & $\{(0.998,1.000),(0.979,1.000)\}$ & $\{(0.989,1.000),(0.897,0.999)\}$ & $\{(0.993,1.000),(0.897,0.999)\}$ \\
$\tau=1$ & $\{(0.984,1.000),(0.962,1.000)\}$ & $\{(1.000,1.000),(0.897,0.999)\}$ & $\{(1.000,1.000),(0.998,1.000)\}$ \\
\hline
\end{tabular}

Table 3

Sensitivity analysis on both values of $\psi$ and $\theta_{1}=1-\theta_{2}$ under $\lambda=0.6$ and $\tau=0.8$

\begin{tabular}{|c|c|c|c|c|c|}
\hline$\psi$ & $\theta 1=1-\theta 2$ & ZOTC & ZOAMS & $\mu T C$ & $\mu A M S$ \\
\hline \multirow{10}{*}{0.1} & 0 & $(4274161.01,4285811.389)$ & $(10108.142,11726.397)$ & $(0.00039,0.016)$ & $(1,1)$ \\
\hline & 0.1 & $(4212922.253,4232331.818)$ & $(10009.17,11705.535)$ & $(0.072,0.862)$ & $(0.862,0.984)$ \\
\hline & 0.2 & (3930183.581, 4212922.253) & $(10009.17,11478.822)$ & $(0.593,0.862)$ & $(0.813,0.862)$ \\
\hline & 0.3 & $(3841895.17,4208251.428)$ & $(10000.273,11388.651)$ & $(0.745,0.916)$ & $(0.745,0.849)$ \\
\hline & 0.4 & $(3791536.219,4208251.428)$ & $(10000.273,11328.095)$ & $(0.831,0.916)$ & $(0.699,0.849)$ \\
\hline & 0.5 & $(3756124.667,4203897.336)$ & $(9978.503,11272.368)$ & $(0.892,0.966)$ & $(0.657,0.819)$ \\
\hline & 0.6 & $(3730714.748,4202874.383)$ & $(9970.925,11217.289)$ & $(0.936,0.978)$ & $(0.615,0.808)$ \\
\hline & 0.7 & $(3711657.4,4201008.507)$ & $(9946.047,11163.992)$ & $(0.969,1)$ & $(0.575,0.774)$ \\
\hline & 0.8 & $(3696884.057,4201008.507)$ & $(9946.047,11103.173)$ & $(0.994,1)$ & $(0.529,0.774)$ \\
\hline & 0.9 & $(3694162.749,4201008.507)$ & $(9946.047,11081.403)$ & $(0.999,1)$ & $(0.512,0.774)$ \\
\hline \multirow{9}{*}{0.2} & 0 & $(4212922.253,4232331.818)$ & $(10009.17,11705.535)$ & $(0.072,0.862)$ & $(0.862,0.984)$ \\
\hline & 0.1 & $(3930183.581,4212922.253)$ & $(10009.17,11478.822)$ & $(0.593,0.862)$ & $(0.813,0.862)$ \\
\hline & 0.2 & $(3841895.17,4212922.253)$ & $(10009.17,11388.651)$ & $(0.745,0.862)$ & $(0.745,0.862)$ \\
\hline & $0.3-0.4$ & $(3841895.17,4208251.428)$ & $(10000.273,11388.651)$ & $(0.745,0.916)$ & $(0.745,0.849)$ \\
\hline & 0.5 & $(3781265.406,4203897.336)$ & $(9978.503,11313.162)$ & $(0.849,0.966)$ & $(0.688,0.819)$ \\
\hline & 0.6 & $(3752950.755,4202874.383)$ & $(9970.925,11266.327)$ & $(0.898,0.978)$ & $(0.652,0.808)$ \\
\hline & 0.7 & $(3711657.4,4202874.383)$ & $(9970.925,11163.992)$ & $(0.969,0.978)$ & $(0.575,0.808)$ \\
\hline & 0.8 & $(3711657.4,4201008.507)$ & $(9946.047,11163.992)$ & $(0.969,1)$ & $(0.575,0.774)$ \\
\hline & 0.9 & $(3696884.057,4201008.507)$ & $(9946.047,11103.173)$ & $(0.994,1)$ & $(0.529,0.774)$ \\
\hline \multirow{8}{*}{0.3} & 0 & $(3908431.61,4212922.253)$ & $(10009.17,11457.8)$ & $(0.63,0.862)$ & $(0.797,0.862)$ \\
\hline & $0.1-0.2$ & $(3841895.17,4212922.253)$ & $(10009.17,11388.651)$ & $(0.745,0.862)$ & $(0.745,0.862)$ \\
\hline & $0.3-0.4$ & $(3841895.17,4208251.428)$ & $(10000.273,11388.651)$ & $(0.745,0.916)$ & $(0.745,0.849)$ \\
\hline & 0.5 & $(3829883.253,4208251.428)$ & $(10000.273,11375.595)$ & $(0.765,0.916)$ & $(0.735,0.849)$ \\
\hline & 0.6 & $(3765419.126,4203897.336)$ & $(9978.503,11288.786)$ & $(0.876,0.966)$ & $(0.669,0.819)$ \\
\hline & 0.7 & $(3748619.643,4202874.383)$ & $(9970.925,11257.072)$ & $(0.905,0.978)$ & $(0.645,0.808)$ \\
\hline & 0.8 & $(3711657.4,4202874.383)$ & $(9970.925,11163.992)$ & $(0.969,0.978)$ & $(0.575,0.808)$ \\
\hline & 0.9 & $(3711657.4,4201008.507)$ & $(9946.047,11163.992)$ & $(0.969,1)$ & $(0.575,0.774)$ \\
\hline \multirow{6}{*}{0.4} & $0-0.3$ & $(3841895.17,4212922.253)$ & $(10009.17,11388.651)$ & $(0.745,0.862)$ & $(0.745,0.862)$ \\
\hline & $0.4-0.5$ & $(3841895.17,4208251.428)$ & $(10000.273,11388.651)$ & $(0.745,0.916)$ & $(0.745,0.849)$ \\
\hline & 0.6 & $(3791536.219,4208251.428)$ & $(10000.273,11328.095)$ & $(0.831,0.916)$ & $(0.699,0.849)$ \\
\hline & 0.7 & $(3765419.126,4203897.336)$ & $(9978.503,11288.786)$ & $(0.876,0.966)$ & $(0.669,0.819)$ \\
\hline & 0.8 & $(3752950.755,4202874.383)$ & $(9970.925,11266.327)$ & $(0.898,0.978)$ & $(0.652,0.808)$ \\
\hline & 0.9 & $(3730714.748,4202874.383)$ & $(9970.925,11217.289)$ & $(0.936,0.978)$ & $(0.615,0.808)$ \\
\hline \multirow{5}{*}{0.5} & $0-0.3$ & $(3841895.17,4212922.253)$ & $(10009.17,11388.651)$ & $(0.745,0.862)$ & $(0.745,0.862)$ \\
\hline & $0.4-0.6$ & $(3841895.17,4208251.428)$ & $(10000.273,11388.651)$ & $(0.745,0.916)$ & $(0.745,0.849)$ \\
\hline & 0.7 & (3829889.185, 4208251.428) & $(10000.273,11375.595)$ & $(0.765,0.916)$ & $(0.735,0.849)$ \\
\hline & 0.8 & $(3781263.282,4203897.336)$ & $(9978.503,11313.162)$ & $(0.849,0.966)$ & $(0.688,0.819)$ \\
\hline & 0.9 & $(3756122.543,4203897.336)$ & $(9978.503,11272.368)$ & $(0.892,0.966)$ & $(0.657,0.819)$ \\
\hline
\end{tabular}


Table 4

Characteristics of the second model

\begin{tabular}{lllll}
\hline $\begin{array}{l}\text { Problem } \\
\text { instance }\end{array}$ & $\begin{array}{l}\text { Number of } \\
\text { Bloodmobiles }\end{array}$ & $\begin{array}{l}\text { Number of } \\
\text { vehicles }\end{array}$ & $\begin{array}{l}\text { Objective } \\
\text { function }\end{array}$ & Solution Representation \\
\hline 1 & 5 & 2 & 339.3765 & $\{3,2,6,4,1,5\}$ \\
2 & 8 & 2 & 284.2587 & $\{8,4,3,1,9,6,7,2,5\}$ \\
3 & 8 & 3 & 276.5026 & $\{5,9,1,3,7,2,10,6,8,4\}$ \\
4 & 10 & 3 & 249.4581 & $\{6,7,8,11,5,4,3,12,2,9,10,1\}$ \\
5 & 14 & 4 & 284.8717 & $\{13,10,12,1,15,7,14,2,6,16,3,11,8,5\}$ \\
6 & 20 & 4 & 333.061 & $\{10,18,6,15,21,1,12,2,5,4,20,22,13,17,19,3,11,23,9,14,7,8,16\}$ \\
7 & 25 & 5 & 305.2511 & $\{11,2,6,3,9,26,1,18,4,23,22,27,10,14,12,19,28,15,17,13,16,7,29,21,5,8,25,24,20\}$ \\
8 & 30 & 5 & 354.3582 & $\{23,28,4,7,9,12,31,15,25,26,30,13,32,10,8,5,14,17,24,1,33,20,2,19,27,29,16,22,34,6$ \\
& & & & $, 3,11,21,18\}$ \\
9 & 40 & 6 & 364.3436 & $\{39,25,27,26,9,40,41,3,31,8,1,30,34,20,12,19,42,2,10,11,17,43,32,22,28,37,36,14,4$ \\
& & & $4,33,35,18,13,23,24,4,6,45,16,38,15,21,7,29,5\}$ \\
\hline
\end{tabular}

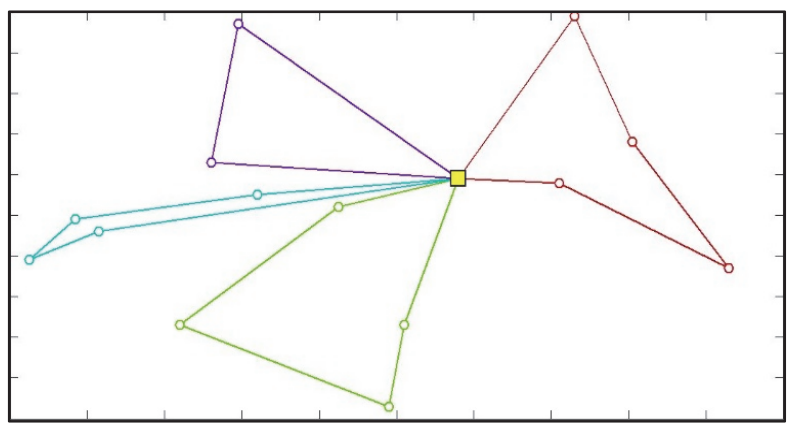

Fig. 7. Solution representation for problem instance 5

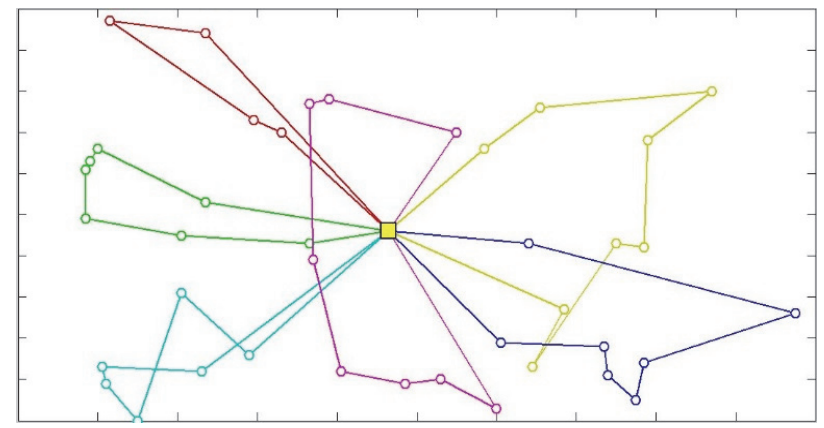

Fig. 8. Solution representation for problem instance 9

\section{Conclusion}

Platelets are the most expensive and perishable blood products. Therefore, many academicians and practitioners have highlighted the importance of platelets supply chain planning to mirror demands and supply. Blood collection planning to produce platelets was studied in this paper. In order to address the problem, two models were proposed. In the first model, location planning of bloodmobiles in the planning horizon was studied. The model seeks to maximize potential blood collection and minimize the associated operational costs. In order to solve the model it is first converted into a crisp multi objective linear programming model by ME approach and then it was solved by TA approach. Several examples have been solved for different values of optimistic pessimistic parameters and various values of TA parameters to demonstrate the impacts of different values on the problem. The second model was for shuttles routing problems which was formulated as a vehicle routing problem with time windows. The problem was solved via simulated annealing approach. Several problem instances with different sizes have been solved to show the applicability of the proposed solution approach. For future researches, one may cope with the uncertainty using mixed fuzzy stochastic programming. Considering multi blood center problem and studying the interaction among them could be another issue. Moreover, applying other meta-heuristic solution techniques may also be considered in future researches.

\section{References}

Beliën, J., \& Forcé, H. (2012). Supply chain management of blood products: A literature review. European Journal of Operational Research, 217(1), 1-16.

Brooks, S. P., \& Morgan, B. J. (1995). Optimization using simulated annealing. The Statistician, 44(2), $241-257$.

Cumming, P. D., Kendall, K. E., Pegels, C. C., Seagle, J. P., \& Shubsda, J. F. (1976). A collections planning model for regional blood suppliers: description and validation. Management Science, 22(9), 962-971.

Dongen, A., Ruiter, R., Abraham, C., \& Veldhuizen, I. (2014). Predicting blood donation maintenance: the importance of planning future donations.Transfusion, 54(3pt2), 821-827.

Fontaine, M. J., Chung, Y. T., Rogers, W. M., Sussmann, H. D., Quach, P., Galel, S. A., ... \& Erhun, F. (2009). Improving platelet supply chains through collaborations between blood centers and transfusion services. Transfusion,49(10), 2040-2047. 
Ghandforoush, P., \& Sen, T. K. (2010). A DSS to manage platelet production supply chain for regional blood centers. Decision Support Systems, 50(1), 32-42.

Haijema, R., van der Wal, J., \& van Dijk, N. M. (2007). Blood platelet production: Optimization by dynamic programming and simulation.Computers \& Operations Research, 34(3), 760-779.

Hemmelmayr, V., Doerner, K. F., Hartl, R. F., \& Savelsbergh, M. W. (2010). Vendor managed inventory for environments with stochastic product usage.European Journal of Operational Research, 202(3), 686-695.

Jiménez, M., Arenas, M., Bilbao, A., \& Rodr1, M. V. (2007). Linear programming with fuzzy parameters: an interactive method resolution.European Journal of Operational Research, 177(3), 1599-1609.

Katsaliaki, K. (2008). Cost-effective practices in the blood service sector.Health policy, 86(2), $276-287$.

Lenstra, J. K. (1997). Local search in combinatorial optimization. Princeton University Press.

Luhandjula, M. K. (2015). Fuzzy optimization: Milestones and perspectives.Fuzzy Sets and Systems, $274,4-11$.

Madden, E., Murphy, E. L., \& Custer, B. (2007). Modeling red cell procurement with both double-red-cell and wholeblood collection and the impact of European travel deferral on units available for transfusion.Transfusion, 47(11), 2025-2037.

Nagurney, A., Masoumi, A. H., \& Yu, M. (2012). Supply chain network operations management of a blood banking system with cost and risk minimization. Computational Management Science, 9(2), 205-231.

Osorio, A. F., Brailsford, S. C., \& Smith, H. K. (2015). A structured review of quantitative models in the blood supply chain: a taxonomic framework for decision-making. International Journal of Production Research, 53(24), 71917212.

Oswalt, R. M. (1977). A review of blood donor motivation and recruitment.Transfusion, 17(2), 123-135.

Pirlot, M. (1992). General local search heuristics in combinatorial optimization: a tutorial. Belgian Journal of Operations Research, Statistics and Computer Science, 32(1-2), 7-69.

Pishvaee, M. S., \& Torabi, S. A. (2010). A possibilistic programming approach for closed-loop supply chain network design under uncertainty.Fuzzy sets and systems, 161(20), 2668-2683.

Rabbani, M., Farrokhi-asl, H., \& Rafiei, H. (2016). A hybrid genetic algorithm for waste collection problem by heterogeneous fleet of vehicles with multiple separated compartments. Journal of Intelligent \& Fuzzy Systems, 30(3), 1817-1830.

Rezaei-Malek, M., Tavakkoli-Moghaddam, R., Zahiri, B., \& Bozorgi-Amiri, A. (2016). An interactive approach for designing a robust disaster relief logistics network with perishable commodities. Computers \& Industrial Engineering,94, 201-215.

Şahinyazan, F. G., Kara, B. Y., \& Taner, M. R. (2015). Selective vehicle routing for a mobile blood donation system. European Journal of Operational Research, 245(1), 22-34.

Selim, H., \& Ozkarahan, I. (2008). A supply chain distribution network design model: an interactive fuzzy goal programming-based solution approach. The International Journal of Advanced Manufacturing Technology, 36(3-4), 401-418.

Torabi, S. A., \& Hassini, E. (2008). An interactive possibilistic programming approach for multiple objective supply chain master planning. Fuzzy Sets and Systems, 159(2), 193-214.

US Department of Health and Human Services. (2011). The 2009 national blood collection and utilization survey report. Washington, DC: US Department of Health and Human Services, Office of the Assistant Secretary for Health, 15.

Van Dijk, N., Haijema, R., Van Der Wal, J., \& Sibinga, C. S. (2009). Blood platelet production: a novel approach for practical optimization. Transfusion,49(3), 411-420.

Veldhuizen, I. J. T., Doggen, C. J. M., Atsma, F., \& De Kort, W. L. A. M. (2009). Donor profiles: demographic factors and their influence on the donor career. Vox sanguinis, 97(2), 129-138.

Veldhuizen, I., Folléa, G., \& Kort, W. (2013). Donor cycle and donor segmentation: new tools for improving blood donor management. Vox sanguinis, 105(1), 28-37.

Wang, R. C., \& Liang, T. F. (2005). Applying possibilistic linear programming to aggregate production planning. International Journal of Production Economics, 98(3), 328-341.

Xu, J., \& Zhou, X. (2013). Approximation based fuzzy multi-objective models with expected objectives and chance constraints: Application to earth-rock work allocation. Information Sciences, 238, 75-95.

Zahraee, S. M., Rohani, J. M., Firouzi, A., \& Shahpanah, A. (2015). Efficiency improvement of blood supply chain system using Taguchi method and dynamic simulation. Procedia Manufacturing, 2, 1-5.

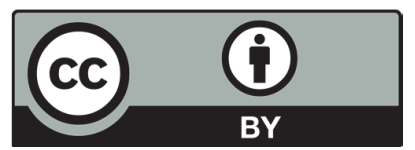

(C) 2016 by the authors; licensee Growing Science, Canada. This is an open access article distributed under the terms and conditions of the Creative Commons Attribution (CCBY) license (http://creativecommons.org/licenses/by/4.0/). 